Yoshihide Kozai

Tokyo Astronomical Observatory, Mitaka, Tokyo 181, Japan

In 1962 the author published a paper on secular perturbations of asteroids with high eccentricity and inclination (Kozai, 1962) and found that for such asteroids the secular perturbations are more complicated than those estimated by the classical linear theory. Before that the author(Kozai, 1954) tried to extend the classical theory to include terms of squares of disturbing masses, eccentricities and inclinations in expressions of secular motions of angular variables and found that the sum of the longitudes of the perihelion and the ascending node is not as stable quantity as the classical theory predicted, and, therefore, it cannot be used to estimate the ages of asteroid families. Then Williams(1969) and Yuasa(1973) extended the classical theory further.

In this paper the author computes the secular perturbations of numbered asteroids and short-periodic comets whose semi-major axes are less than $30 \mathrm{a} \cdot \mathrm{u}$. by assuming that (1) the disturbing planets are Jupiter, Saturn, Uranus and Neptune, (2) their orbital semi-major axes are not mucl disturbed, and (3) the disturbing planets are moving along circular orbits on Jupiter's orbital plane. According to the assumption (2) any asteroid or comet with commensurable mean motion and any comet which can approach to any disturbing planet very closely should be excluded.

According to the assumption (2) the mean anomaly of the disturbed body and the mean longitude of each of the disturbing planets can be eliminated from the disturbing function by averaging it with respect to each of the angular variables independently. The averaging is carried out not by any analytical way but by a numerical harmonic analysis with $5^{\circ}$ as one step for the variables. After the elimination the semi-major axis can be assumed to be constant. By the assumption (3) the longitude of the ascending node of the disturbed body does not appear in the averaged disturbing function when the orbital plane of Jupiter is adopted as the reference $\mathrm{plane}$, and, therefore, the $z$-component of the angular momentum, $\left(a\left(1-e^{2}\right)\right)^{1 / 2} \cos i$, is conserved, where $i$ is the inclination with respect to Jupiter's orbital plane. And as the averaged Hamiltonian as we11 as the disturbing function do not depend on time explicitly, the Hamiltonian $F$ is conserved. 
Now the system of the equations of motion is reduced to that of one degree of freedom, and it is the equation for the secular perturbations. The variables are $G=\left(a\left(1-e^{2}\right)\right)^{1 / 2}$, the angular momentum, and $g$, the argument of perihelion, and the Hamiltonian is regarded as a function of $x$ $=\left(1-e^{2}\right) 1 / 2$ and $2 \mathrm{~g}$ with $\mathrm{a}$ and $\mathrm{H}=\mathrm{x} \cos \mathrm{i}$ as parameters. Therefore, $\mathrm{x}$ can be solved as a function of $2 \mathrm{~g}$, in fact, a periodic function of $2 \mathrm{~g}$, and as $\mathrm{H}$ is given, both the eccentricity and the inclination can be graphically expressed as functions of $\mathrm{g}$. As the values of a and $\mathrm{H}$ are given for each of the asteroids and the comets, the values of the Hamiltonian can be computed for various sets of $x$ and $g$, and then equi-F-value curves are drawn in $\mathrm{g}-\mathrm{x}$ plane, where $\mathrm{g}$-axis extends from $0^{\circ}$ to $90^{\circ}$ and $\mathrm{x}$-axis extends from $\mathrm{H}$ to 1 . There are symmetries with respect to $\mathrm{g}=0^{\circ}, 90^{\circ}, 180^{\circ}$ and $270^{\circ}$. Two lines, $\mathrm{x}=1$ and $\mathrm{x}=\mathrm{H}$, are always equi-F-value curves and $\mathrm{x}=1$ corresponds to a circular orbit whereas $\mathrm{x}=\mathrm{H}$ corresponds to an orbit on $\mathrm{Ju}-$ piter's orbital plane. The two orbits are not disturbed due to the secular perturbation unless the argument of perihelion is of libration. As $\mathrm{H}$ is decreased, the variation of $\mathrm{x}$ becomes wider, and if the value of $\mathrm{H}$ is below a certain value around 0.8 , a libration region appears in the $g-$ $x$ diagram. If the value of $F$ corresponding to the concerned asteroid or comet is that in the libration region, the argument of perihelion does not make a complete revolution but makes a libration around $90^{\circ}$ or $270^{\circ}$.

Except for the libration case the eccentricity of any of the asteroids takes its minimum and the inclination takes its maximum at $\mathrm{g}=0^{\circ}$ and $180^{\circ}$ and vice versa at $\mathrm{g}=90^{\circ}$ and $270^{\circ}$. For the libration case both maximum and minimum take place at $90^{\circ}$ or $270^{\circ}$. Therefore, the orbits become the least eccentric when the perihelion is on Jupiter's orbital plane and can avoid any very close approach to Jupiter if the orbit is inside of Jupiter. For most of the asteroids it is found that $\mathrm{x}$ is larger than $\cos i$ and for such cases the variation of the eccentricity is wider than that of the inclination. In Figure 1 the maximum, the minimum and the present values of the eccentricities and the inclinations of eight asteroids belonging to a family, Pallas family by Hirayama(1928) and family No. 28 by Brouwer(1951), are plotted with asteroid numbers. For each of the asteroids a narrow libration region appears in the $g-x$ diagram as the valuses of $\mathrm{H}$ are nearly equal to 0.8 , although for non of them the argument of perihelion is of libration. In the figure the crosses in the upper left

i

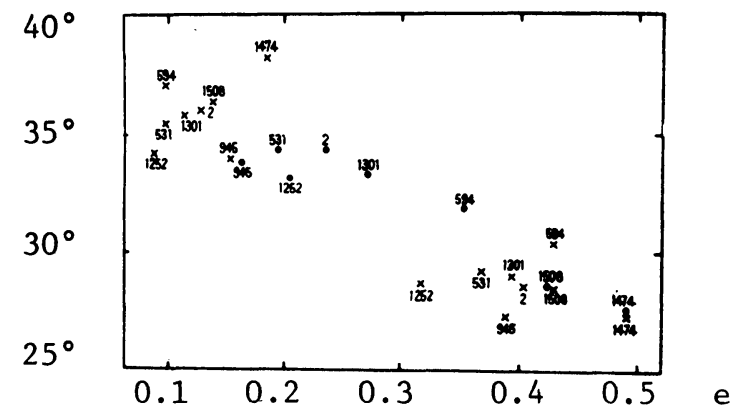

Figure 1. Variations of the eccentricities and the inclinations for asteroids belonging to Pallas family. 
corner correspond to the values of the eccentricity and the inclination at $\mathrm{g}=0^{\circ}$ and $180^{\circ}$ and those in the lower right corner correspond to those at $\mathrm{g}=90^{\circ}$ and $270^{\circ}$. The circles correspond to the present values. For these asteroids the eccentricities change by more than 0.3 and the inclinations change by $10^{\circ}$ as functions of the argument of perihelion. Differences between the proper eccentricities and inclinations and osculating ones are much smaller than the variations. The asteroids 1474 and 1508 were not included in this family in Brouwer's paper, however, it is found that they are real members of the family.

There are four asteroids among the numbered ones, for which the arguments of perihelion are of libration. They are 944 Hidalgo, 1373 Cincinnati, 1866 Sisyphus and 1981. Kozai(1962) found that the argument of perihelion of Cincinnati $\left(a=3.411\right.$ a.u.) is of libration between $69^{\circ}$ and $111^{\circ}$ by a simpler computation. The eccentricity and the inclination change between 0.318 and 0.559 and between $40 .^{\circ} 2$ and $29 .^{\circ} 2$, respectively, as one sees in Figure 2, where the scales of the ordinate are not given in $x$ but in $e$ and $i$ although it is scaled with equal interval of $x$. The present place is marked with the cross on one of the equi-F-value curves. Because of the libration both the perihelion and the aphelion of this asteroid cannot be placed on Jupiter's orbital plane, and, therefore, it cannot approach to Jupiter very closely even though the aphelion distance can become larger than the semi-major axis of Jupiter. There are three asteroids, for which the minimum aphelion distances are larger than 4.4 a.u. One of them is this asteroid, and the other two belong to Hecuba group. For the latter two the critical arguments are of libration around $0^{\circ}$ (Franklin et al., 1975) and, therefore, they can avoid any close approach to Jupiter.

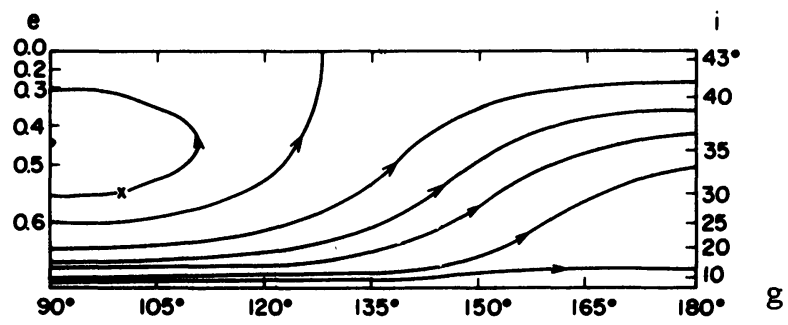

Figure 2. The $g-x$ diagram for 1373 Cincinnati.

According to the present computation the argument of perihelion is of libration for a peculiar asteroid, Hidalgo, $(a=5.82$ a.u. $)$ between

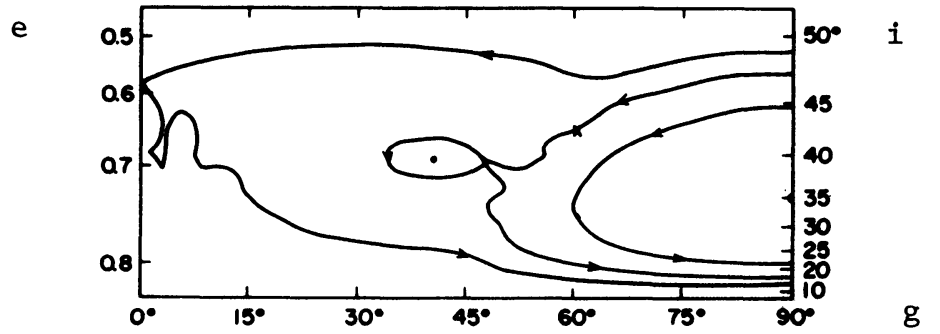

Figure 3. A part of the g-x diagram for 944 Hidalgo. 
$48^{\circ}$ and $132^{\circ}$, and the eccentricity and the inclination change between 0 . 568 and 0.811 and between $47 .{ }^{\circ} 3$ and $17 .^{\circ} 2$, respectively, whereas the present values are $e=0.658$ and $i=42 .^{\circ} 26$. Though the argument of perihelion is now decreasing from $59 .^{\circ} 5$, after reaching to $48^{\circ}$ it will increase and when it will become $90^{\circ}$ the perihelion distance will be as small as 1.1 a.u. Then the orbit will be quite similar to that of a comet. As Hidalgo has a possibility to approach very closely to Jupiter, the present computations might have some errors. However, Nakano's numerical integration results(1977) suggest that the errors are not so large. For the other two asteroids, for which the arguments of perihelion are of libration, the semi-major axes are 1.89 and $1.78 \mathrm{a.u}$. and their orbits are quite similar to those of comets and can avoid any very close approach to Mars. Besides the four asteroids there appear librations regions in $\mathrm{g}-\mathrm{x}$ diagrams for 23 asteroids.

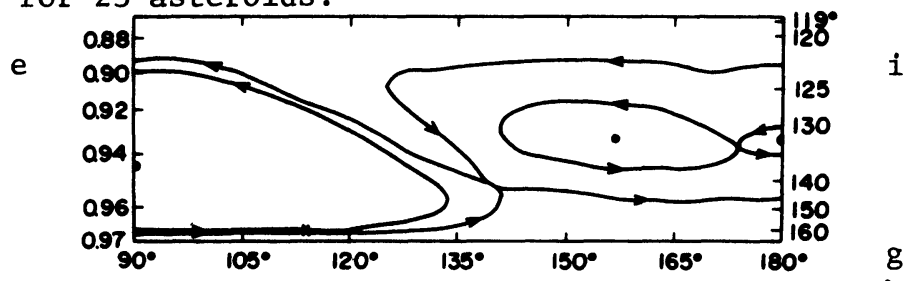

Figure 4. A part of the $g-x$ diagram for Halley's comet. The present place is marked by the cross which is on one of the equ-Fvalue curves.

The situation is quite contrary for short-periodic comets. of 104 comets treated here 78 comets have their semi-major axes less than that of Jupiter. Of them 68 comets have libration regions in their $\mathrm{g}-\mathrm{x}$ diagrams because the values of $\mathrm{H}$ are usually small. However, for none of them the argument of perihelion is of libration. For most of them $x$ is less than cos $i$ and the variation of the inclination is wider than that of the eccentricity. However, of the short-periodic comets the semimajor axes of 26 comets are between that of Jupiter and $30 \mathrm{a} . \mathrm{u}$. Of the 26 comets 25 have libration regions in $\mathrm{g}-\mathrm{x}$ diagrams, the only one exception being $\mathrm{P} /$ Schwassma-n-Wachmann $I$. And the arguments of perihelion are of libration for 15 comets. However, even for the libration cases the eccentricities do not change so much as those of some asteroids. For Halley's comet, for an example, the argument of perihelion is of libration between $47^{\circ}$ and $133^{\circ}$ and the eccentricity and the inclination change between 0.899 and 0.968 and between $123 .{ }^{\circ} 3$ and $164 .{ }^{\circ} 4$, respectively. The present values are $e=0.967, i=161 .{ }^{\circ} 2$ and $g=114 .{ }^{\circ} 4$ which is increasing. Therefore, the eccentricity of this comet is now nearly maximum and the perihelion distance will not decrease in near future. However, for this short range of the variation the perihelion distance is changed by $1.2 \mathrm{a.u}$. which is large enough to affect the evolution of the comet. Also there are a few comets, for which the eccentricities are changed in wider ranges like Hidalgo.

Since the eccentricity and the inclination do change very much for asteroids with small values of $\mathrm{H}$, the proper eccentricity and inclination which are derived by the classical linear theory are not adequate to clas- 


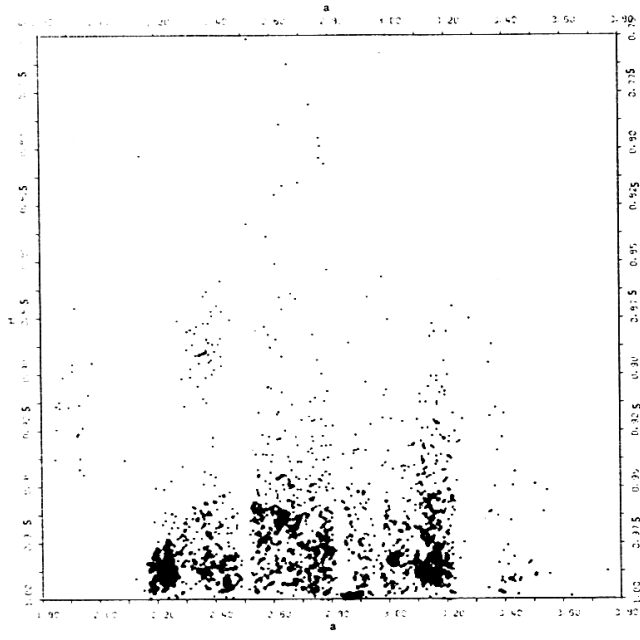

Figure 4. a-H diagram.

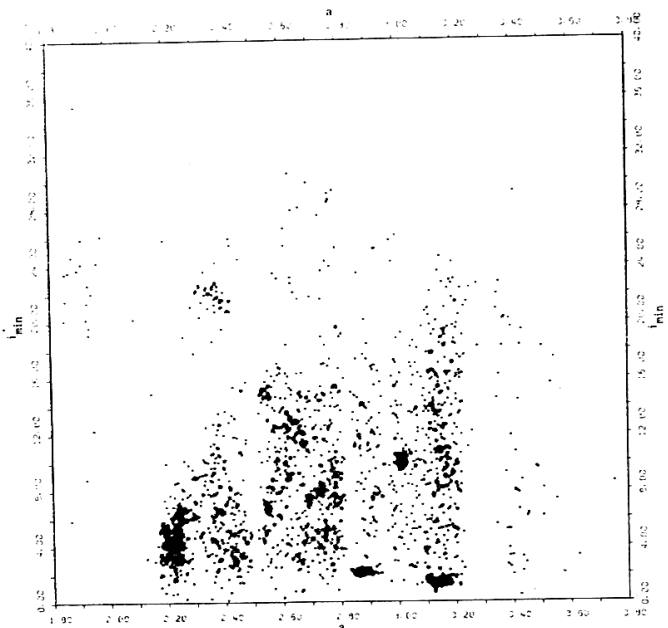

Figure $5 \cdot{ }^{a-i_{m i n}}$ diagram.

sify such asteroids into families. The author believes that the minimum value of the inclination(or the maximum value or the eccentricity) and $H$ as well as the semi-major axis are more adequate and stable quantities which can be used for classification of the asteroids with not only small values of $\mathrm{H}$ but also $\mathrm{H}$ nearly equal to 1 . In Figures 4 and 5 the numbered asteroids are plotted in $\mathrm{a}-\mathrm{H}$ plane and $\mathrm{a}-\mathrm{i}_{\min } \mathrm{plane}$, respectively. Even in these figures 6 famous families discovered by Hirayama(1923 and 1928) as well as the family in Figure 1 clearly show up. More clear pictures can be obtained when the asteroids are plotted in a-H-i min space. By this way the seven families in the following table are derived. In the table the names of the families after Hirayama(1923 and 1928), the numbers of their members by the present analysis, Hirayama(1928) and Brouwer(1951) in this order, and the mean values of the semi-major axes, $H$ and the minimum inclinations as well as their mean deviations are given. Also the mean values of the maximum eccentricities are given for a reference.

\begin{tabular}{|c|c|c|c|c|c|c|c|c|c|}
\hline Name & \multicolumn{3}{|c|}{ Numbers } & a & & $\mathrm{H}$ & & $i_{m i}$ & e \\
\hline Themis & 77 & 32 & 53 & $3.140 \pm$ & 0.024 & $0.9881 \pm$ & 0.0033 & $1 .^{\circ} 40+0 .^{\circ} 21$ & 0.108 \\
\hline Eos & 89 & 27 & 58 & $3.015 \pm$ & 0.007 & $0.9821 \mp$ & 0.0013 & 10. $01 \mp 0.27$ & 0.052 \\
\hline Coronis & 48 & 20 & 33 & $2.875 \pm$ & 0.018 & $0.9984 \mp$ & 07 & 2. $14 \mp 0.14$ & 0.030 \\
\hline Maria & 20 & 14 & 17 & $2.543 \mp$ & 0.014 & $0.9609 \mp$ & 0.0023 & 14. $93 \mp 0.30$ & 0.074 \\
\hline Flora & 228 & 63 & 125 & $2.229 \mp$ & 0.032 & $0.9860 \mp$ & 0.0060 & 4. $58 \mp 1.40$ & 0.104 \\
\hline Phocaea & 34 & 11 & 21 & $2.371 \mp$ & 0.033 & $382 \mp$ & 0.0093 & 21. $85 \mp 0.60$ & 0.207 \\
\hline Pallas & 8 & 3 & 6 & $2.723 \pm$ & 0.057 & $0.8023 \pm$ & 0.0167 & 32. $16 \pm 2.43$ & 0.229 \\
\hline
\end{tabular}

As one can see, some families are very compact whereas others are not. The scattering of $\mathrm{H}$ is small for the families whose eccentricities are small. This may suggest that the neglecting the eccentricities of the disturbing planets gives stronger effects on more eccentric asteroids such as those belonging to Phocaea and Pallas families. The effects are very small for those belonging to Eos, Coronis and Maria families. Even 
though these effects are taken into account, there are still some loose families such as Flore and Pallas. Therefore, the author has an idea that these loose families were not originated from single mother asteroids as the other compact ones were probably originated from single ones. Probably many asteroids were pushed out from regions where secular commensurabilities exist and gathered there as loose families as Williams( 1969) pointed out. In fact several gaps seen in Figures 4 and 5 are situated near regions of secular commensurablity.

The computations were made with the computor FACOM 230-58 at the Computing Center of the Tokyo Astronomical Observatory by the orbital elements of the asteroids and comets provided by $\mathrm{Dr}$. K. Hurukawa.

\section{REFERENCES .}

Brouwer, D.: 1951, "Astron. J." 56, pp.9-32.

Franklin, F. A. et al.: 1975, "Astron. J." 80, pp.729-746.

Hirayama, K.: 1918, "Astron. J." 31, pp. 185-188.

Hirayama, K.: 1923, "Japan J. Astron. Geophys." 1, pp. 55-93.

Hirayama, K.: 1928, "Japan J. Astron. Geophys." 5, pp. 137-162.

Kozai, Y.: 1954, "Publ. Astron. Soc. Japan" 6, pp. 41-66.

Kozai, Y.: 1962, "Astron. J." 67, pp.591-598.

Nakano, K.: 1977, "Private Communication".

Williams, J. G.: 1969, "Secular Perturbations in the Solar System", Dissertation, University of California, Los Angeles, U.S.A.

Yuasa, M.: 1973, "Pub1. Astron. Soc. Japan" 25, pp. 399-446.

\section{DISCUSSION}

Kiang: 1. Did I understand that comet Halley is a librator?

2. Hirayama and later Brouwer use proper $e, \underline{i}$ and $\underline{a}$ to find families. What are your defining parameters? Do you get better defined families? Have you found any new families?

3. Would not the functional relation between $e$ and $i$ in your simplified model not explain the elongated form of the families in the proper (e, $\underline{1})$-plane?

Kozai: 1. Yes, it is, according to my computation. However, as you can see in Fig. 4 the equi-F-value curve for Halley's comet is just inside a limiting curve and, therefore, if there is a small error in my computation, it is possible that the argument of perihelion is not of libration.

2. My parameters are $a, 1-e^{2} \cos i$, and the minimum values of i. I can get better defined families at least for the Pallas case. 3. No. 
Marsden: Don't you think there is a significant difference between (1373) Cincinnati and the other objects for which you now find argument-of-perihelion libration? The libration of (1373) very successfully keeps this object away from Jupiter, and the orbit is thus rather stable. The comets and the other minor planets for which you find libration all make close approaches either to Jupiter or to the Earth, so it would seem that these librations can have little effect on the stability of the orbits.

Kozai: The two asteroids can avoid any very close approach to Mars due to librations. 